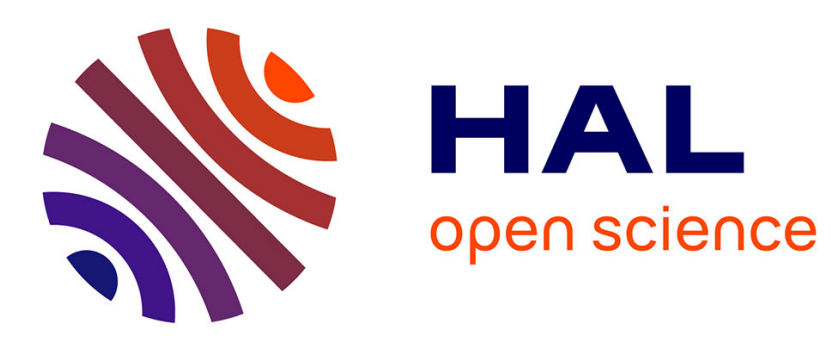

\title{
Homogenization of fronts in highly heterogeneous media
}

Guy Barles, Annalisa Cesaroni, Matteo Novaga

\section{To cite this version:}

Guy Barles, Annalisa Cesaroni, Matteo Novaga. Homogenization of fronts in highly heterogeneous media. SIAM Journal on Mathematical Analysis, 2011, 43 (1), pp.212-227. hal-00494583

\section{HAL Id: hal-00494583 \\ https://hal.science/hal-00494583}

Submitted on 23 Jun 2010

HAL is a multi-disciplinary open access archive for the deposit and dissemination of scientific research documents, whether they are published or not. The documents may come from teaching and research institutions in France or abroad, or from public or private research centers.
L'archive ouverte pluridisciplinaire HAL, est destinée au dépôt et à la diffusion de documents scientifiques de niveau recherche, publiés ou non, émanant des établissements d'enseignement et de recherche français ou étrangers, des laboratoires publics ou privés. 


\title{
Homogenization of fronts in highly heterogeneous media
}

\author{
Guy Barles* Annalisa Cesaroni ${ }^{\dagger} \quad$ Matteo Novaga $^{\dagger}$
}

\begin{abstract}
We consider the evolution by mean curvature in a highly heterogeneous medium, modeled by a periodic forcing term, with large $L^{\infty}$-norm but with zero average. We prove existence of a homogenization limit, when the dimension of the periodicity cell tends to zero, and show some properties of the effective velocity.
\end{abstract}

Key-words : Homogenization, propagation of fronts, heterogeneous media, evolution by mean curvature, viscosity solutions

AMS subject classifications : 35B27, 35K55, 35J20, 53C44, 49L25

\section{Introduction}

This paper deals with mean curvature flow in a heterogeneous medium, represented by a $\mathbb{Z}^{n}$ periodic function $g \in \operatorname{Lip}\left(\mathbb{R}^{n}\right)$ acting on the flow as an additive forcing term. This problem has been already considered in the literature, starting from the papers [12, 19. In particular, in [14, 10] the authors consider the following scaling

$$
V^{\varepsilon}(x)=\varepsilon H(x)+g\left(\frac{x}{\varepsilon}\right) \quad \text { for } x \in \Gamma^{\varepsilon}(t),
$$

where $\Gamma^{\varepsilon}(t)$ is the evolving hypersurface, $V^{\varepsilon}(x)$ is its normal velocity at $x \in \Gamma^{\varepsilon}(t)$, and $H(x)$ its mean curvature. Under appropriate assumptions on the function $g$, one can prove that the evolution law (1) converges, as $\varepsilon \rightarrow 0$, to the first-order anisotropic law

$$
V(x)=c(\nu(x)) \quad \text { for } x \in \Gamma(t),
$$

where $\Gamma(t)$ denotes the limit of $\Gamma^{\varepsilon}(t), V(x)$ the limiting normal velocity at $x \in \Gamma(t)$ and $c$ is a continuous function of the normal vector $\nu(x)$ to $\Gamma(t)$ at $x$, and can be determined by solving a suitable cell problem.

Since $c=0$ when $g$ has zero average, a natural question is what happens if we consider a different time-scaling of (1), namely

$$
V^{\varepsilon}(x)=H(x)+\frac{1}{\varepsilon} g\left(\frac{x}{\varepsilon}\right) \quad \text { for } x \in \Gamma^{\varepsilon}(t),
$$

under the additional assumption that $g$ has zero average.

The object of the present paper is the study of the limit of the evolution law (2) as $\varepsilon \rightarrow 0$. We assume that the evolving hypersurface $\Gamma^{\varepsilon}(t)$ is a graph of a function $u^{\varepsilon}(\cdot, t)$ with respect to a

* Laboratoire de Mathématiques et Physique Théorique (UMR CNRS 6083). Fédération Denis Poisson (FR CNRS 2964) Université de Tours. Faculté des Sciences et Techniques, Parc de Grandmont, 37200 Tours, France, email: barles@lmpt.univ-tours.fr

${ }^{\dagger}$ Dipartimento di Matematica Pura e Applicata, Università di Padova, via Trieste 63, 35121 Padova, Italy, email: acesar@math.unipd.it, novaga@math.unipd.it 
fixed hyperplane, independent of $\varepsilon$, and that the forcing term $g$ does not depend on the variable orthogonal to such hyperplane. In this case, the function $u^{\varepsilon}$ satisfies the equation

$$
u_{t}^{\varepsilon}(t, x)=\operatorname{tr}\left[\left(\mathbf{I}-\frac{D u^{\varepsilon} \otimes D u^{\varepsilon}}{1+\left|D u^{\varepsilon}\right|^{2}}\right) D^{2} u^{\varepsilon}\right]+\frac{1}{\varepsilon} g\left(\frac{x}{\varepsilon}\right) \sqrt{1+\left|D u^{\varepsilon}\right|^{2}} \quad \text { in }(0,+\infty) \times \mathbb{R}^{n},
$$

where we require that the initial datum $u_{0}: \mathbb{R}^{n} \rightarrow \mathbb{R}$ is a uniformly continuous function such that $u_{0}(x)-q x$ is bounded for some $q \in \mathbb{R}^{n}$.

Another motivation for considering (3) comes from the following homogenization problem:

$$
u_{t}^{\varepsilon}=\operatorname{tr}\left[\left(\mathbf{I}-\frac{D u^{\varepsilon} \otimes D u^{\varepsilon}}{1+\left|D u^{\varepsilon}\right|^{2}}\right) D^{2} u^{\varepsilon}\right]+g\left(\frac{x}{\varepsilon}\right) \sqrt{1+\left|D u^{\varepsilon}\right|^{2}} \quad \text { in }(0,+\infty) \times \mathbb{R}^{n} .
$$

Since the function $g$ is bounded, one can show that, as $\varepsilon \rightarrow 0, u^{\varepsilon} \rightarrow u$ locally uniformly in $\mathbb{R}^{n} \times[0,+\infty)$. Moreover, $u$ solves the limit equation

$$
u_{t}=\operatorname{tr}\left[\left(\mathbf{I}-\frac{D u \otimes D u}{1+|D u|^{2}}\right) D^{2} u\right]+\left(\int_{[0,1]^{n}} g d x\right) \sqrt{1+|D u|^{2}} \quad \text { in }(0,+\infty) \times \mathbb{R}^{n} .
$$

This result, derived in [11] when $n=1$, can be obtained in general dimensions by the so-called perturbed test function method [15], which is by now a standard tool in viscosity solutions theory applied to homogenization problems. More precisely, one considers the formal asymptotic expansion

$$
u^{\varepsilon}(x, t)=u(x, t)+\varepsilon^{2} \chi\left(\frac{x}{\varepsilon}, x, t\right)
$$

where the corrector $\chi(\xi, x, t)=\psi(\xi, D u(x, t))$ is periodic in $\xi$ and solves, for every fixed $p=$ $D u(x, t)$, the cell problem

$$
\operatorname{tr}\left[\left(\mathbf{I}-\frac{p \otimes p}{1+|p|^{2}}\right) D_{\xi \xi}^{2} \chi\right]=\left(\int_{[0,1]^{n}} g(x) d x-g(\xi)\right) \sqrt{1+|p|^{2}} \text { in } \mathbb{R}^{n} .
$$

Plugging the expansion (6) in (值) and using the comparison principle for viscosity solutions, one obtains that $u$ solves (5).

In this paper, we apply the same method to Equation (3), and we prove that, under a suitable assumption on the function $g$ (see Section 2), the limit function $u$ solves the anisotropic parabolic equation

$$
u_{t}=\operatorname{tr}\left(A(D u) D^{2} u\right) \quad \text { in }(0,+\infty) \times \mathbb{R}^{n},
$$

where $A(p)$ is a smooth function depending on $g$, with values in the set of positive definite symmetric matrices. Obviously, when $g \equiv 0$ we have $A(p)=\mathbf{I}-\frac{p \otimes p}{1+|p|^{2}}$.

When $n=1$, thanks to an explicit representation formula for $A(p)$, we can further show that

$$
0<A(p) \leq \frac{1}{1+p^{2}} \quad \text { for all } p \in \mathbb{R}
$$

and that $\lim _{|p| \rightarrow \infty} A(p)\left(1+p^{2}\right)=0$, when $g \not \equiv 0$. In particular, this implies that the presence of $g$ has the effect of decreasing the speed of the front in the limit, without stopping the motion.

A further consequence of our result is the nonexistence of compact embedded solutions of the prescribed curvature problem

$$
H+g=0,
$$

for all $g$ such that $A(p) \neq 0$ for some $p \in \mathbb{R}^{n}$. For similar results in the nonperiodic setting, we refer to [18] and references therein.

The paper is organized as follows. In Section 2 we describe the standing assumptions on the forcing term $g$. Section 3 and 4 are devoted to the analysis of two ergodic problems in $\mathbb{R}^{n}$, which permit to define the limit parabolic operator $F(p, X)=\operatorname{tr}(A(p) X)$. In Section 4 we consider the case of planar curves (i.e. $n=1$ ), and obtain a more explicit description of the function $A(p)$ (see Proposition 4.1). Finally, Section 5 contains the main homogenization result (see Theorem 5.3) and a discussion of its consequences for the related prescribed curvature problem (7). 


\section{Standing assumptions}

In this section we state some conditions on the forcing term $g$, which will hold throughout the paper. Setting $Q:=(0,1)^{n}$, our first condition is:

(G1) $g: \mathbb{R}^{n} \rightarrow \mathbb{R}$ is Lipschitz continuous, $\mathbb{Z}^{n}$-periodic and $\int_{Q} g(y) d y=0$.

The requirement that $g$ has zero average on $Q$ is necessary due to the homogenization result for Equations (1) and (1) discussed in the Introduction.

We also need a condition ensuring that the oscillation of $g$ on $Q$ is not too large. Let us first define the space $B V_{\text {per }}(Q)$ of functions which have periodic bounded variation in $Q$. We refer to [3] for a general introduction to functions of bounded variation and sets of finite perimeter.

It is a classical result that any $u \in B V(Q)$ admits a trace $u^{Q}$ on $\partial Q$ (see e.g. [3, Thm 3.87]). Let $\partial_{0} Q:=\partial Q \cap\left\{y: \prod_{i=1}^{n} y_{i}=0\right\}$ and let $\sigma: \partial_{0} Q \rightarrow \partial Q$ be the function $\sigma(y):=y+\sum_{i=1}^{n} \lambda_{i}(y) e_{i}$, where $\lambda_{i}(y)=1$ if $y_{i}=0$ and $\lambda_{i}(y)=0$ otherwise. The periodic total variation of $u \in B V(Q)$ is defined as

$$
|D u|_{\text {per }}(Q):=|D u|(Q)+\int_{\partial_{0} Q}\left|u^{Q}(y)-u^{Q}(\sigma(y))\right| d \mathcal{H}^{n-1}(y) .
$$

The space $B V_{\text {per }}(Q)$ is the space $B V(Q)$ endowed with the norm

$$
\|u\|_{B V_{\mathrm{per}}(Q)}:=\|u\|_{L^{1}(Q)}+|D u|_{\mathrm{per}}(Q) .
$$

For every $E \subseteq Q$ we define the periodic perimeter of $E$ as

$$
\operatorname{Per}_{\mathrm{per}}(E, Q):=\left|D \chi_{E}\right|_{\mathrm{per}}(Q)
$$

where $\chi_{E}$ is the characteristic function of $E$. We observe that $B V_{\text {per }}(Q)$ coincides with $B V\left(\mathbb{T}^{n}\right)$, where $\mathbb{T}^{n}:=\mathbb{R}^{n} / \mathbb{Z}^{n}$ is the $n$-dimensional torus. In particular, the following Coarea Formula holds [3, Thm 3.40]

$$
|D u|_{\text {per }}(Q)=\int_{\mathbb{R}} \operatorname{Per}_{\text {per }}(\{u>t\}, Q) d t \quad \text { for all } u \in B V_{\text {per }}(Q) .
$$

The second condition we assume on $g$ is:

(G2) there exists $\delta<1$ such that for every $E \subseteq Q$ of finite perimeter

$$
\int_{E} g(y) d y \leq \delta \operatorname{Per}_{\mathrm{per}}(E, Q) .
$$

Note that, when $n>1$, condition (G2) is satisfied whenever $\|g\|_{L^{n}(Q)}<C(Q)$, where $C(Q)$ is the isoperimetric constant of $\mathbb{T}^{n}$. Indeed, since $g$ has zero average, possibly exchanging $E$ with $Q \backslash E$ in (11) we can assume that $|E| \leq 1 / 2$. By the isoperimetric inequality on $\mathbb{T}^{n}$ [22], we then obtain

$$
\int_{E} g(y) d y \leq\|g\|_{L^{n}(Q)}|E|^{\frac{n-1}{n}} \leq \frac{\|g\|_{L^{n}(Q)}}{C(Q)} \operatorname{Per}_{\mathrm{per}}(E, Q)=\delta \operatorname{Per}_{\mathrm{per}}(E, Q)
$$

where $\delta=\|g\|_{L^{n}(Q)} / C(Q)<1$.

When $n=1$, condition (G2) is equivalent to

$$
\max _{y \in[0,1]} \int_{0}^{y} g(s) d s-\min _{y \in[0,1]} \int_{0}^{y} g(s) d s<2
$$

which is the same condition assumed in [10]. 


\section{The cell problems and the effective operator}

In this section, we consider two ergodic problems in $Q$, see (13), (15), whose solutions are useful to define the limit problem as $\varepsilon \rightarrow 0$ of the singularly perturbed Equations (3).

Lemma 3.1 Under the standing assumptions, for every $p \in \mathbb{R}^{n}$ the equation

$$
-\operatorname{div}\left(\frac{D \chi+p}{\sqrt{1+(D \chi+p)^{2}}}\right)=g(y) \quad \text { in } \mathbb{R}^{n}
$$

admits a $\mathbb{Z}^{n}$-periodic solution $\chi(y ; p) \in \mathcal{C}^{2+\alpha}\left(\mathbb{R}^{n}\right)$, for all $\alpha<1$, which is unique up to an additive constant. Moreover $\chi$ depends smoothly on $p$.

Proof We observe that (13) is the Euler-Lagrange equation of the functional

$$
J_{p}(u):=\int_{Q}\left(\sqrt{1+(D u+p)^{2}}-g u\right) d y+\int_{\partial_{0} Q}\left|u^{Q}(y)-u^{Q}(\sigma(y))\right| d \mathcal{H}^{n-1}(y)
$$

which is a convex lower semicontinuous functional on $B V_{\text {per }}(Q)$ (see [3]).

We claim that $J_{p}$ is coercive on the subspace

$$
B V_{\mathrm{per}}^{0}(Q):=\left\{u \in B V_{\mathrm{per}}(Q): \int_{Q} u=0\right\} .
$$

Notice that $|D u|_{\text {per }}(Q)$ is an equivalent norm on $B V_{\text {per }}^{0}(Q)$ [3, Thm 3.44]. By condition (G2) and the Coarea Formula (10), recalling that $\int_{Q} g d y=0$, we have

$$
\int_{Q} g u d y=\int_{\mathbb{R}} \int_{\{u>t\}} g d y d t \leq \delta \int_{\mathbb{R}} \operatorname{Per}_{\mathrm{per}}(\{u>t\}, Q) d t=\delta|D u|_{\mathrm{per}}(Q),
$$

for all $u \in B V_{\text {per }}(Q)$. Therefore, we obtain

$$
\begin{aligned}
J_{p}(u) & \geq \int_{Q}|D u+p|-\int_{Q} g u d y+\int_{\partial_{0} Q}\left|u^{Q}(y)-u^{Q}(\sigma(y))\right| d \mathcal{H}^{n-1}(y) \\
& \geq-|p|-\int_{Q} g u d y+|D u|_{\mathrm{per}}(Q) \\
& \geq-|p|+(1-\delta)|D u|_{\mathrm{per}}(Q),
\end{aligned}
$$

which proves that $J_{p}$ is coercive on $B V_{\text {per }}^{0}(Q)$.

Since $J_{p}$ is coercive and lower semicontinuous on $B V_{\mathrm{per}}^{0}(Q)$, it admits a minimizer $\chi(\cdot ; p)$. Moreover, by convexity, $\chi(\cdot ; p)$ is the unique minimizer of $J_{p}$ on $B V_{\text {per }}(Q)$, up to an additive constant.

Finally, by [16], any solution $\chi(y ; p)$ of (13) is Lipschitz continuous, and hence of class $\mathcal{C}^{2+\alpha}\left(\mathbb{R}^{n}\right)$ by standard elliptic regularity [17. Also, by differentiation of (12) in the $p$-variables, the same regularity holds for any derivative of $\chi(y ; p)$ with respect to $p$.

REMARK Notice that the periodic function $\psi_{p}(y)=\chi(y ; p)+p \cdot y$ solves the prescribed curvature problem

$$
-\operatorname{div}\left(\frac{D \psi_{p}}{\sqrt{1+\left(D \psi_{p}\right)^{2}}}\right)=g(y), \quad y \in \mathbb{R}^{n} .
$$

In particular, the graph of $\psi_{p}$ is a plane-like solution of the geometric equation $H=g$, lying at a bounded distance from the hyperplane $\left\{(y, p \cdot y): y \in \mathbb{R}^{n}\right\}$. We refer to [9] for a general analysis of such solutions. 
Lemma 3.2 For any $p \in \mathbb{R}^{n}$ and $M \in \mathbf{S}_{n}$ there exists a unique constant $F(p, M)$ such that there exists a $\mathbb{Z}^{n}$-periodic solution $\psi(y ; p, M) \in \mathcal{C}^{2+\alpha}\left(\mathbb{R}^{n}\right)$, for all $\alpha<1$, to the cell problem

$$
\begin{aligned}
& F(p, M)=\operatorname{tr}\left[\left(\mathbf{I}-\frac{\left(p+D_{y} \chi\right) \otimes\left(p+D_{y} \chi\right)}{1+\left|p+D_{y} \chi\right|^{2}}\right)\left(D^{2} \psi+M+2 D_{p y}^{2} \chi M\right)\right] \\
& -2\left(p+D_{y} \chi\right)^{T} D_{y y}^{2} \chi\left(\frac{D \psi+\left(D_{p} \chi\right)^{T} M}{1+\left|p+D_{y} \chi\right|^{2}}-\frac{\left(p+D_{y} \chi\right) \cdot\left(D \psi+\left(D_{p} \chi\right)^{T} M\right)}{\left(1+\left|p+D_{y} \chi\right|^{2}\right)^{2}}\left(p+D_{y} \chi\right)\right) \\
& +g(y) \frac{\left(p+D_{y} \chi\right) \cdot\left(D \psi+\left(D_{p} \chi\right)^{T} M\right)}{\sqrt{1+\left|p+D_{y} \chi\right|^{2}}},
\end{aligned}
$$

where $\chi(y ; p)$ is the solution to (13) with $\chi(0 ; p)=0$. Moreover $\psi(y ; p, M)$ is in $\mathcal{C}^{2}\left(\mathbb{R}^{n}\right)$ and is unique up to an additive constant.

Finally, there exists a $n \times n$ symmetric matrix $A(p)$, depending smoothly on $p$, such that

$$
F(p, M)=\operatorname{tr}(A(p) M) .
$$

Proof Observe that, using the fact that $\chi$ solves (13), Equation (15) can be rewritten as

$$
\begin{aligned}
\operatorname{tr}\left(B(y, p) D^{2} \psi\right)+b(y, p) \cdot D \psi=F(p, M) & -\operatorname{tr}\left[B(y, p)\left(\mathbf{I}+2 D_{p y}^{2} \chi\right) M\right] \\
& -(b(y, p))^{T} M D_{p} \chi,
\end{aligned}
$$

where $B(y, p):=\mathbf{I}-\frac{\left(p+D_{y} \chi\right) \otimes\left(p+D_{y} \chi\right)}{1+\left|p+D_{y} \chi\right|^{2}}$ is a symmetric, positive definite matrix and

$$
b(y, p):=-\frac{2\left(p+D_{y} \chi\right)^{T} D_{y y}^{2} \chi}{1+\left|p+D_{y} \chi\right|^{2}}+\left[\frac{3 g(y)}{\sqrt{1+\left|p+D_{y} \chi\right|^{2}}}+\frac{2 \Delta_{y y} \chi}{1+\left|p+D_{y} \chi\right|^{2}}\right]\left(p+D_{y} \chi\right) .
$$

Moreover, recalling that $\chi$ is $\mathbb{Z}^{n}$-periodic and smooth, we get that (17) is a uniformly elliptic equation and that both $B(y, p)$ and $b(y, p)$ are $\mathbb{Z}^{n}$-periodic in $y$. The existence of a unique constant $F(p, M)$ such that (17) admits a continuous periodic solution $\psi$ is then a well-known fact (see 国, Thm II.2], [1, Thm 7.1]). Finally, $\psi$ is unique up to an additive constant and is of class $\mathcal{C}^{2+\alpha}$ with respect to $y$, for all $\alpha<1$, by elliptic regularity [17].

We also have an explicit characterization of $F(p, M)$. Indeed, consider the differential operator

$$
\mathcal{L}_{p}(\phi):=\operatorname{tr}\left(B(y, p) D^{2} \phi\right)+b(y, p) \cdot D \phi
$$

and let $\mathcal{L}_{p}^{\star}$ be its formal adjoint. Then, the equation $\mathcal{L}_{p}^{\star}(m)=0$ admits a $\mathbb{Z}^{n}$-periodic solution $m(y ; p)>0$, which is unique up to a multiplicative constant (see [7] Thm II.4.2]), so that we may fix $\int_{Q} m(y ; p) d y=1$. Notice that $m(y ; p)$, as well as $\chi(y ; p)$, depends smoothy on $p$ by elliptic regularity.

In [7, Thm II.6.1] (see also [15, Thm 2.1], [1, Corollary 6.2]) it is proved that

$$
F(p, M)=\int_{Q}\left[\operatorname{tr}\left(B(y, p)\left(\mathbf{I}+2 D_{p y}^{2} \chi(y ; p)\right) M\right)+(b(y, p))^{T} M D_{p} \chi(y ; p)\right] m(y ; p) d y .
$$

This formula implies, in particular, the regularity of $F$ with respect to $p$, since the functions $b(y, \cdot)$ and $B(y, \cdot)$ are smooth, due to the regularity properties of $\chi$ and $m$.

Finally, the above representation formula for $F$ also implies $(16)$, since $F(p, \cdot)$ is a linear function of $M$ for any fixed $p$, and hence which can be written $\operatorname{as} \operatorname{tr}[A(p) M]$ for some symmetric matrix $A(p)$.

REMARK Notice that, always by elliptic regularity, from (15) it follows that the map $g \mapsto A(p)$ (when defined) is continuous with respect to the Lipschitz norm of $g$. In particular, since $A(p)=$ I $-\frac{p \otimes p}{1+|p|^{2}}$ when $g=0$, we get that there exists $\delta>0$ such that, if $\|g\|_{\text {Lip }}<\delta$, then $A(0) \neq 0$. Notice that, possibly reducing $\delta$, this condition implies (11). 


\section{The effective operator in dimension 1}

When $n=1$, we obtain a much more explicit description of the effective operator $F(p, M)$ (see Proposition 4.1). This is done by solving explicitly the two cell problems (13) and (15).

Lemma 4.1 Under the standing assumptions, for every $p \in \mathbb{R}$ the periodic solution to (13) such that $\chi(0 ; p)=0$ is given by

$$
\chi(y ; p)=-p y+\int_{0}^{y} \frac{c(p)-G(s)}{\sqrt{1-(c(p)-G(s))^{2}}} d s,
$$

where $G^{\prime}(y)=g(y), \int_{0}^{1} G(y) d y=0$, and $c=f^{-1}$ with $f(c)=\int_{0}^{1} \frac{c-G(y)}{\sqrt{1-(c-G(y))^{2}}} d y$.

Proof We denote by $\bar{m}:=\max _{[0,1]} G$ and $\underline{m}=\min _{[0,1]} G$.

Integrating once the equation, easy computations show that every solution to 13 ) satisfies $\frac{1}{1+\left(p+\chi_{y}\right)^{2}}=$ $1-(c-G(y))^{2}$ for some constant $c$. Therefore solving the problem necessarily requires $\max _{[0,1]}(G-$ $c)<1$ and $\min _{[0,1]}(G-c)>-1$. It is possible to find $c$ satisfying this condition if and only if $\bar{m}-\underline{m}<2$. This is ensured by (11). In this case it is sufficient to choose $c \in(\bar{m}-1, \underline{m}+1)$. For such constants $c$, we get that $\chi_{y}(y)=-p+\frac{c-G(y)}{\sqrt{1-(c-G(y))^{2}}}$. We define the function $f:(\bar{m}-1, \underline{m}+1) \rightarrow \mathbb{R}$ by

$$
f(c):=\int_{0}^{1} \frac{c-G(y)}{\sqrt{1-(c-G(y))^{2}}} d y .
$$

Straightforward computations show that $f$ is strictly increasing and we claim that $f(\bar{m}-1, \underline{m}+1)=$ $(-\infty,+\infty)$. Indeed we remark that, by assumption $(\mathrm{G} 1), G \in \mathcal{C}^{1,1}(\mathbb{R})$ and, if $y_{0}$ is a maximum point of $G$, then for $y$ close to $y_{0}$ we have $\left|G(y)-G\left(y_{0}\right)\right| \leq k\left|y-y_{0}\right|^{2}$. As a consequence, if $c=\bar{m}-1$, in a small neighborhood of $y_{0}$ we have

$1-(\bar{m}-1-G(y))^{2}=1-\left(G\left(y_{0}\right)-1-G(y)\right)^{2}=2\left(G(y)-G\left(y_{0}\right)\right)+\left(G(y)-G\left(y_{0}\right)\right)^{2} \leq \tilde{k}\left|y-y_{0}\right|^{2}$, for some constant $\tilde{k}>0$. Possibly changing the constant $\tilde{k}$, we then get

$$
\frac{c-G(y)}{\sqrt{1-(c-G(y))^{2}}} \leq-\frac{\tilde{k}}{\left|y-y_{0}\right|} .
$$

This inequality shows that the function is not integrable for $c=\bar{m}-1$ and therefore $f(c) \rightarrow-\infty$ as $c \rightarrow \bar{m}-1$. An analogous argument holds when $c=\underline{m}+1$.

Since we are looking for periodic solutions to Equation (13), we impose the condition $\int_{0}^{1} \chi_{y}(y) d y=0$. This gives $f(c)=p$, that is, $c=f^{-1}$.

Notice that the function $c$ is smooth and

$$
c^{\prime}(p)=\frac{1}{\int_{0}^{1}\left[1-(c(p)-G(y))^{2}\right]^{-\frac{3}{2}} d y} .
$$

Proposition 4.1 Under the standing assumptions and for $n=1$, the function $A(p)$ in Lemma 3.7 is given by

$$
0<A(p)=\frac{c^{\prime}(p)}{\int_{0}^{1} \sqrt{1-(c(p)-G(y))^{2}} d y} \leq \frac{1}{1+p^{2}} \quad \forall p \in \mathbb{R},
$$

where $G(y)$ and $c(p)$ are as in Lemma 4.1. Moreover if $g \not \equiv 0$ there exists a constant $K_{g}$ such that

$$
0<A(p)\left(1+p^{2}\right) \leq \frac{K_{g}}{\sqrt{1+p^{2}}}
$$

In particular $A(p)\left(1+p^{2}\right) \rightarrow 0$ as $|p| \rightarrow+\infty$. 
Proof To obtain the characterization of $A$, we explicitly solve Equation (15). Due to the homogeneity properties of (15) with respect to $M$, we immediately get $A(p)=F(p, 1)$. We rewrite (15) with $M=1$. We consider the coefficient of $\psi_{y}$ and obtain, recalling the characterization (18) of $\chi$,

$$
\begin{aligned}
& \frac{g(y)\left(p+\chi_{y}\right)}{\sqrt{1+\left(p+\chi_{y}\right)^{2}}}-\frac{2 \chi_{y y}\left(p+\chi_{y}\right)}{\left(1+\left(p+\chi_{y}\right)^{2}\right)^{2}} \\
= & \frac{p+\chi_{y}}{1+\left(p+\chi_{y}\right)^{2}}\left[g(y) \sqrt{1+\left(p+\chi_{y}\right)^{2}}-\frac{2 \chi_{y y}}{1+\left(p+\chi_{y}\right)^{2}}\right]=3 g(y)(c(p)-G(y)) .
\end{aligned}
$$

The last two terms on the right-hand side of (15) coincide with

$$
\frac{2 \chi_{y y} \chi_{p}\left(p+\chi_{y}\right)}{\left(1+\left(p+\chi_{y}\right)^{2}\right)^{2}}-\frac{g(y) \chi_{p}\left(p+\chi_{y}\right)}{\sqrt{1+\left(p+\chi_{y}\right)^{2}}}=-3 g(y)(c(p)-G(y)) \chi_{p} .
$$

Then, using the explicit formula for $\chi_{y p}$ deduced from (18), (15) can be rewritten as

$$
\psi_{y y}+\chi_{y p}+\frac{3 g(y)(c(p)-G(y))}{1-(c(p)-G(y))^{2}}\left(\psi_{y}+\chi_{p}\right)=\frac{F(p, 1)}{1-(c(p)-G(y))^{2}}-\frac{c^{\prime}(p)}{\left(1-(c(p)-G(y))^{2}\right)^{\frac{3}{2}}}
$$

Note that $\left.\frac{3 g(y)(c(p)-G(y))}{1-(c(p)-G(y))^{2}}=\left[\log \left(1-(c(p)-G(y))^{2}\right)^{\frac{3}{2}}\right)\right]_{y}$. Therefore we obtain

$$
\left[\left(1-(c(p)-G(y))^{2}\right)^{\frac{3}{2}}\left(\psi_{y}+\chi_{p}\right)\right]^{\prime}=F(p, 1) \sqrt{1-(c(p)-G(y))^{2}}-c^{\prime}(p)
$$

and integrating we get, for some constant $d(p)$,

$$
\left(1-(c(p)-G(y))^{2}\right)^{\frac{3}{2}}\left(\psi_{y}+\chi_{p}\right)=d(p)+F(p, 1) \int_{0}^{y} \sqrt{1-(c(p)-G(s))^{2}} d s-c^{\prime}(p) y .
$$

Then

$$
\psi_{y}=-\chi_{p}+\frac{d(p)+F(p, 1) \int_{0}^{y} \sqrt{1-(c(p)-G(s))^{2}} d s-c^{\prime}(p) y}{\left(1-(c(p)-G(y))^{2}\right)^{\frac{3}{2}}} .
$$

We look for a periodic solution $\psi$, then we impose that $\psi_{y}(0)=\psi_{y}(1)$. Recalling the formula for $c^{\prime}(p)$ and $\chi_{p}$, we get $\chi_{p}(0)=\chi_{p}(1)=0$, so $\psi_{y}(0)=\frac{d(p)}{\left(1-(c(p)-G(0))^{2}\right)^{\frac{3}{2}}}$ and

$$
\psi_{y}(1)=\frac{d(p)+F(p, 1) \int_{0}^{1} \sqrt{1-(c(p)-G(s))^{2}} d s-c^{\prime}(p)}{\left(1-(c(p)-G(1))^{2}\right)^{\frac{3}{2}}} .
$$

Then, since $G$ is periodic, we obtain the condition $F(p, 1) \int_{0}^{1} \sqrt{1-(c(p)-G(y))^{2}} d y=c^{\prime}(p)$, which gives the desired characterization (19) of $A(p)$. In particular, recalling the definition of $c^{\prime}(p)$ we get that $A(p)>0$ for every $p$.

We prove now that $A(p)\left(1+p^{2}\right) \leq 1$. By definition of $c(p)$ and Hölder inequality we get

$$
|p| \leq \int_{0}^{1}\left|\frac{c(p)-G(y)}{\sqrt{1-(c(p)-G(y))^{2}}}\right| d y \leq\left[\int_{0}^{1} \frac{(c(p)-G)^{2}}{1-(c(p)-G(y))^{2}} d y\right]^{\frac{1}{2}} .
$$

Therefore

$$
1+p^{2} \leq \int_{0}^{1} \frac{1}{1-(c(p)-G(y))^{2}} d y
$$

By Holder inequality we get

$$
\begin{aligned}
\int_{0}^{1} \frac{1}{1-(c(p)-G(y))^{2}} d y & \leq\left[\int_{0}^{1} \frac{1}{\left[1-(c(p)-G(y))^{2}\right]^{\frac{3}{2}}} d y\right]^{\frac{2}{3}} \\
& =\frac{\left[\int_{0}^{1} \frac{1}{\left[1-(c(p)-G(y))^{2}\right]^{\frac{3}{2}}} d y\right]^{-\frac{1}{3}}}{c^{\prime}(p)} .
\end{aligned}
$$


Again by Jensen and Holder inequalities we obtain

$$
\begin{aligned}
\frac{1}{\int_{0}^{1} \sqrt{1-(c(p)-G(y))^{2}} d y} & \leq \int_{0}^{1} \frac{1}{\sqrt{1-(c(p)-G(y))^{2}}} d y \\
& \leq\left[\int_{0}^{1} \frac{1}{\left[1-(c(p)-G(y))^{2}\right]^{\frac{3}{2}}} d y\right]^{\frac{1}{3}} .
\end{aligned}
$$

Finally, recalling the definition (19) of $A(p)$ (21), (22) and (23) give $1+p^{2} \leq \frac{1}{A(p)}$.

Finally we prove (20). From inequalities (21) and (22), we get

$$
c^{\prime}(p) \leq\left(\frac{1}{1+p^{2}}\right)^{\frac{3}{2}} .
$$

We define the function

$$
h(p):=\left(\int_{0}^{1} \sqrt{1-(c(p)-G(y))^{2}} d y\right)^{-1} .
$$

Notice that $h^{\prime}(p)=c^{\prime}(p) p h^{-2}(p)$ and then $h$ is decreasing in $(-\infty, 0)$ and increasing in $(0,+\infty)$. Moreover

$$
\begin{aligned}
& \lim _{p \rightarrow+\infty} h(p)=\left(\int_{0}^{1} \sqrt{1-(1+\underline{m}-G(y))^{2}} d y\right)^{-1} \\
& \lim _{p \rightarrow-\infty} h(p)=\left(\int_{0}^{1} \sqrt{1-(1-\bar{m}-G(y))^{2}} d y\right)^{-1} .
\end{aligned}
$$

We define the constant

$$
K_{g}:=\max \left(\frac{1}{\int_{0}^{1} \sqrt{1-(1+\underline{m}-G(y))^{2}} d y}, \frac{1}{\int_{0}^{1} \sqrt{1-(-1+\bar{m}-G(y))^{2}} d y}\right) .
$$

Note that $K_{g}>0$ depends only on $g$ and that it explodes as $g \rightarrow 0$. So we conclude, recalling (19).

REMark Note that if $g \equiv 0$, then $c(p)=\frac{p}{\sqrt{1+p^{2}}}$ and

$$
A(p)=\frac{c^{\prime}(p)}{\int_{0}^{1} \sqrt{1-\frac{p^{2}}{1+p^{2}}} d y}=\frac{\sqrt{1+p^{2}}}{\left(1+p^{2}\right)^{\frac{3}{2}}}=\frac{1}{1+p^{2}} .
$$

Moreover, it is clear that the constant $K_{g}$ in 20 necessarily explodes as $g \rightarrow 0$.

\section{The convergence result}

In this section we study the asymptotic behaviour as $\varepsilon \rightarrow 0$ of the solutions $u^{\varepsilon}$ of the singularly perturbed equations

$$
u_{t}^{\varepsilon}(t, x)=\operatorname{tr}\left[\left(\mathbf{I}-\frac{D u^{\varepsilon} \otimes D u^{\varepsilon}}{1+\left|D u^{\varepsilon}\right|^{2}}\right) D^{2} u^{\varepsilon}\right]+\frac{1}{\varepsilon} g\left(\frac{x}{\varepsilon}\right) \sqrt{1+\left|D u^{\varepsilon}\right|^{2}},
$$

for $(t, x) \in(0,+\infty) \times \mathbb{R}^{n}$, with initial data $u^{\varepsilon}(0, x)=u_{0}(x)$, where

$$
u_{0}(x)=q \cdot x+v_{0}(x) \quad \text { with } v_{0} \text { bounded and uniformly continuous. }
$$


We show that the functions $u^{\varepsilon}$ converge locally uniformly to a function $u$, which is a continuous viscosity solution to the effective quasilinear parabolic equation

$$
u_{t}(t, x)=\operatorname{tr}\left[A(D u(t, x)) D^{2} u(t, x)\right] \quad \text { in }(0,+\infty) \times \mathbb{R}^{n}
$$

with initial datum $u(0, x)=u_{0}(x)$, where the differential operator $F(p, M)=\operatorname{tr}[A(p) M]$ is the one defined in Lemma 3.2. Moreover $u$ is the unique viscosity solution of (26) in the class

$$
\mathcal{L}_{q}:=\left\{u \in \mathcal{C}\left([0,+\infty), R^{n}\right) \text { s.t. } u(t, x)-q \cdot x \in \mathcal{C}_{b}\left([0,+\infty) \times \mathbb{R}^{n}\right)\right\},
$$

where $\mathcal{C}_{b}\left([0,+\infty), \mathbb{R}^{n}\right)$ is the space of bounded continuous functions in $[0,+\infty) \times \mathbb{R}^{n}$. We also discuss the geometric counterpart of this result and some consequences for a related prescribed curvature problem.

We start recalling two comparison principles for solutions of degenerate parabolic equations, which we will apply to the singularly perturbed and to the effective problem.

Theorem 5.1 Let $w^{\varepsilon}, v^{\varepsilon}$ be respectively an upper semicontinuous subsolution and a lower semicontinuous supersolution to (24) in $[0,+\infty) \times \mathbb{R}^{n}$. Assume that there exists a constant $k>0$ such that $\frac{w^{\varepsilon}(t, x)}{1+|x|^{k}}, \frac{v^{\varepsilon}(t, x)}{1+|x|^{k}} \rightarrow 0$ as $|x| \rightarrow+\infty$ uniformly with respect to $t \in[0,+\infty)$, and that $w^{\varepsilon}(0, x) \leq u_{0}(x) \leq v^{\varepsilon}(0, x)$ for every $x \in \mathbb{R}^{n}$, where $u_{0}$ satisfies (25). Then $w^{\varepsilon}(t, x) \leq v^{\varepsilon}(t, x)$ for every $(t, x) \in[0,+\infty) \times \mathbb{R}^{n}$.

Moreover, there exists a unique continuous viscosity solution $u^{\varepsilon}$ in $\mathcal{L}_{q}$ to (24), with initial datum $u_{0}$.

The proof of this comparison principle is given in [5, Theorem 2.1], while the existence of a unique solution to (24) can be done (with easy modifications) as in [5, Cor 2.1].

Notice that, if the initial datum $u_{0}$ is of class $\mathcal{C}^{2+\alpha}\left(\mathbb{R}^{n}\right)$ for some $\alpha \in(0,1)$, then parabolic regularity theory [20] gives that the solutions $u^{\varepsilon}$ in Theorem 5.1 are uniformly of class $\mathcal{C}^{1+\alpha / 2,2+\alpha}([0,+\infty) \times$ $\left.\mathbb{R}^{n}\right)$.

Theorem 5.2 Let $w, v$ be respectively a bounded upper semicontinuous subsolution and a bounded lower semicontinuous supersolution to $u_{t}=\operatorname{tr}\left[\tilde{A}(D u) D^{2} u\right]$ in $[0,+\infty) \times \mathbb{R}^{n}$, where $\tilde{A}(p)$ is a symmetric $n \times n$ matrix, which depends smoothly on $p$ and such that $\tilde{A}(p) \geq 0$ for any $p$. Assume that $w(0, x) \leq v(0, x)$ for every $x \in \mathbb{R}^{n}$. Then $w(t, x) \leq v(t, x)$ for every $(t, x) \in[0,+\infty) \times \mathbb{R}^{n}$.

For the proof we refer to [13].

We will apply this result to the limiting problem $(26)$, letting $\tilde{A}(p):=A(p+q)$ and changing the solution $u(t, x)$ in $\tilde{u}(t, x)=u(t, x)-q \cdot x$. Since we look for solutions in $\mathcal{L}_{q}$, we actually have to deal with bounded solutions $\tilde{u}$.

However, we need to show that the effective differential operator $F(p, M)$ is regular and degenerate elliptic. Surprisingly the degenerate ellipticity of $F$ is not known a priori, and we obtain it in a step of the convergence proof. We point out here that the degenerate ellipticity is expected as a consequence of results of Alvarez, Guichard, Lions and Morel [2] (see also [8]). Indeed, Equation (24) defines a monotone semi-group (in the sense that the solution $u^{\varepsilon}$ depends on $u_{0}$ or $v_{0}$ in a monotone way by the comparison principle) and the limiting semigroup is also expected to be monotone, hence, by the results of [2] or [8], it is certainly associated to a parabolic equation. We finally remark that we can see this monotonicity as a geometric "inclusion principle" and use as well the geometric version of the above results by Souganidis and the first author [6].

We now state our main result.

Theorem 5.3 Let $u^{\varepsilon}$ be the unique continuous viscosity solution to (24) with polynomial growth and initial datum $u_{0}$, which satisfies (25) for some $q \in \mathbb{R}^{n}$. Then $u^{\varepsilon}(t, x)$ converges locally uniformly to the unique function $u$ in the class $\mathcal{L}_{q}$ which solves (26) in the viscosity sense, with initial datum $u(0, x)=u_{0}(x)$. 
Proof The argument of the proof is an appropriate adaptation of the perturbed test function method, introduced by Evans in [15] (see also 12, 10]). As we already pointed out above, we emphasize the fact that we do not know a priori that the limiting equation is degenerate parabolic. The proof is divided into five steps.

Step 1 (Local equiboundedness of $u^{\varepsilon}$.) The existence and uniqueness of $u^{\varepsilon}$ is assured by Theorem 5.1. Actually, it is possible to show that $u^{\varepsilon}$ inherits the same growth of the initial data $u_{0}$. We consider the solution $\chi$ of (13) with $p=q$ and such that $\chi(0 ; q)=0$. Then the function $w^{\varepsilon}(t, x)=$ $\varepsilon \chi\left(\frac{x}{\varepsilon} ; q\right)+q \cdot x+\left\|v_{0}\right\|_{\infty}+\varepsilon\|\chi\|_{\infty}$, where $v_{0}$ is the function appearing in (25), is a stationary solution to (24) with $w^{\varepsilon}(0, x) \geq u_{0}(x)$, for $\varepsilon>0$. Analogously $v^{\varepsilon}(t, x)=\varepsilon \chi\left(\frac{x}{\varepsilon} ; q\right)+q \cdot x-\left\|v_{0}\right\|_{\infty}-\varepsilon\left\|_{\chi}\right\|_{\infty}$ is a stationary solution to (24) with $v^{\varepsilon}(0, x) \leq u_{0}(x)$, for $\varepsilon>0$. Then, by the comparison principle (Theorem 5.1) we obtain that $v^{\varepsilon}(t, x) \leq u^{\varepsilon}(t, x) \leq w^{\varepsilon}(t, x)$, which gives in particular that $\left|u^{\varepsilon}(t, x)-q \cdot x\right| \leq\left\|v_{0}\right\|_{\infty}+2 \varepsilon\|\chi\|_{\infty}$ for any $\varepsilon>0$ and $(t, x) \in[0,+\infty) \times \mathbb{R}^{n}$.

Step 2 (Relaxed semilimits of $u^{\varepsilon}$.) We define the relaxed semilimits (see [13])

$$
\underline{u}(t, x):=\liminf _{\left(\varepsilon, t^{\prime}, x^{\prime}\right) \rightarrow(0, t, x)} u^{\varepsilon}\left(t^{\prime}, x^{\prime}\right), \quad \bar{u}(t, x):=\limsup _{\left(\varepsilon, t^{\prime}, x^{\prime}\right) \rightarrow(0, t, x)} u^{\varepsilon}\left(t^{\prime}, x^{\prime}\right)
$$

for $(t, x) \in[0,+\infty) \times \mathbb{R}^{n}$. Observe that $\bar{u}, \underline{u}$, due to the previous step, satisfy $|\bar{u}(t, x)-q \cdot x| \leq\left\|v_{0}\right\|_{\infty}$, $|\underline{u}(t, x)-q \cdot x| \leq\left\|v_{0}\right\|_{\infty}$ for any $(t, x) \in[0,+\infty) \times \mathbb{R}^{n}$.

We are going to prove that $\bar{u}$ (resp. $\underline{u}$ ) is a viscosity subsolution (resp. supersolution) to (26).

We describe the argument just for $\bar{u}$, since for $\underline{u}$ it is completely analogous. We consider a smooth function $\phi$ and we assume that $\bar{u}-\phi$ has a strict maximum at $(\bar{t}, \bar{x})$; we have to prove that

$$
\phi_{t}(\bar{t}, \bar{x}) \leq \operatorname{tr}\left[A(D \phi(\bar{t}, \bar{x})) D^{2} \phi(\bar{t}, \bar{x})\right] .
$$

We define the perturbed test function

$$
\phi^{\varepsilon}(t, x):=\phi(t, x)+\varepsilon \chi\left(\frac{x}{\varepsilon} ; D \phi(t, x)\right)+\varepsilon^{2} \psi\left(\frac{x}{\varepsilon} ; D \phi(\bar{t}, \bar{x}), D^{2} \phi(\bar{t}, \bar{x})\right),
$$

where $\chi(y ; D \phi(t, x))$ is the periodic solution to (13) with $p=D \phi(t, x)$ and $\chi(0 ; p)=0$, and $\psi\left(y ; D \phi(\bar{t}, \bar{x}), D^{2} \phi(\bar{t}, \bar{x})\right)$ is the periodic solution to $(15)$ with $p=D \phi(\bar{t}, \bar{x}), M=D^{2} \phi(\bar{t}, \bar{x})$ and $\psi(0 ; p, M)=0$. Observe that

$$
\limsup _{\left(\varepsilon, t^{\prime}, x^{\prime}\right) \rightarrow(0, t, x)}\left(u^{\varepsilon}\left(t^{\prime}, x^{\prime}\right)-\phi^{\varepsilon}\left(t^{\prime}, x^{\prime}\right)\right)=\bar{u}(t, x)-\phi(t, x) .
$$

By a standard compactness argument, there exist subsequences $\varepsilon_{n} \rightarrow 0$ and $\left(t_{n}, x_{n}\right) \rightarrow(\bar{t}, \bar{x})$ such that $u^{\varepsilon_{n}}\left(t_{n}, x_{n}\right)-\phi^{\varepsilon_{n}}\left(t_{n}, x_{n}\right) \rightarrow \bar{u}(\bar{t}, \bar{x})-\phi(\bar{t}, \bar{x})$ and $\left(t_{n}, x_{n}\right)$ is a strict maximum of $u^{\varepsilon_{n}}-\phi^{\varepsilon_{n}}$. Since $u^{\varepsilon_{n}}$ is a subsolution to (24), at $\left(t_{n}, x_{n}\right)$ we have

$$
\phi_{t}^{\varepsilon_{n}} \leq \operatorname{tr}\left[\left(\mathbf{I}-\frac{D \phi^{\varepsilon_{n}} \otimes D \phi^{\varepsilon_{n}}}{1+\left|D \phi^{\varepsilon_{n}}\right|^{2}}\right) D^{2} \phi^{\varepsilon_{n}}\right]+\frac{1}{\varepsilon_{n}} g\left(\frac{x_{n}}{\varepsilon_{n}}\right) \sqrt{1+\left|D \phi^{\varepsilon_{n}}\right|^{2}} .
$$

Recalling the definition of $\phi^{\varepsilon}$ in 28 and using standard asymptotic expansion arguments, we can rewrite the r.h.s. of the previous inequality as

$$
\begin{aligned}
& \frac{1}{\varepsilon_{n}}\left\{\operatorname{tr}\left[\left(\mathbf{I}-\frac{(D \phi+D \chi)) \otimes(D \phi+D \chi)}{1+|D \phi+D \chi|^{2}}\right) D^{2} \chi\right]+g\left(\frac{x_{n}}{\varepsilon}\right) \sqrt{1+|D \phi+D \chi|^{2}}\right\} \\
+ & \operatorname{tr}\left[\left(\mathbf{I}-\frac{(D \phi+D \chi) \otimes(D \phi+D \chi)}{1+\left|D \phi+D_{y} \chi\right|^{2}}\right)\left(D^{2} \psi+D^{2} \phi+2 D_{p y}^{2} \chi D^{\phi}\right)\right] \\
- & 2(D \phi+D \chi)^{T} D^{2} \chi\left(\frac{D \psi+\left(D_{p} \chi\right)^{T} D^{2} \phi}{1+|D \phi+D \chi|^{2}}\right) \\
+ & 2(D \phi+D \chi)^{T} D^{2} \chi(D \phi+D \chi) \frac{(D \phi+D \chi) \cdot\left(D \psi+\left(D_{p} \chi\right)^{T} D^{2} \phi\right)}{\left(1+|D \phi+D \chi|^{2}\right)^{2}} \\
+ & g\left(\frac{x_{n}}{\varepsilon}\right) \frac{(D \phi+D \chi) \cdot\left(D \psi+\left(D_{p} \chi\right)^{T} D^{2} \phi\right)}{\sqrt{1+|D \phi+D \chi|^{2}}}+R\left(\varepsilon_{n}\right)
\end{aligned}
$$


where $R\left(\varepsilon_{n}\right) \rightarrow 0$ uniformly as $\varepsilon_{n} \rightarrow 0$. Using the characterization of $\chi$ and $\psi$ as solutions of (13) and (15) respectively, and the regularity of $\phi, \chi, \psi, A$, we obtain that

$$
\phi_{t}\left(t_{n}, x_{n}\right) \leq \operatorname{tr}\left[\left(A\left(D \phi\left(t_{n}, x_{n}\right)\right)\right) D^{2} \phi\left(t_{n}, x_{n}\right)\right]+R^{\prime}\left(\varepsilon_{n}\right)
$$

for some $R^{\prime}\left(\varepsilon_{n}\right) \rightarrow 0$ uniformly as $\varepsilon_{n} \rightarrow 0$. Letting $n \rightarrow+\infty$, we obtain (27).

Step 3 (Degenerate ellipticity of the limiting operator.) We prove the following

Lemma 5.1 The differential operator $F(p, M)=\operatorname{tr}[A(p) M]$ defined in Lemma 3.2 is degenerate elliptic.

Proof It is sufficient to show that the matrix $A(p)$ is nonnegative definite for any $p \in \mathbb{R}^{n}$. We consider the unique solution $u^{\varepsilon}$ to (24) with polynomial growth and initial data $u_{0}(x)=p \cdot x$ (see Theorem 5.1) and the solution $\chi(y ; p)$ of (13) with $\chi(0 ; p)=0$. Then, for all $\varepsilon>0$, the function $w^{\varepsilon}(t, x)=\varepsilon \chi\left(\frac{x}{\varepsilon} ; p\right)+p \cdot x+\varepsilon\|\chi\|_{\infty}$ is a stationary solution to (24) with $w^{\varepsilon}(0, x) \geq u_{0}(x)$. Analogously $v^{\varepsilon}(t, x)=\varepsilon \chi\left(\frac{x}{\varepsilon} ; p\right)+p \cdot x-\varepsilon\|\chi\|_{\infty}$ is a stationary solution to 24) with $v^{\varepsilon}(0, x) \leq u_{0}(x)$, for $\varepsilon>0$. By the comparison principle (Theorem 5.1) we then obtain that $v^{\varepsilon}(t, x) \leq u^{\varepsilon}(t, x) \leq$ $w^{\varepsilon}(t, x)$, which gives in particular $\lim _{\varepsilon \rightarrow 0} u^{\varepsilon}(t, x)=p \cdot x$, locally uniformly.

According to Step 2, if $(\bar{t}, \bar{x})$ is a strict maximum of $u(t, x)-\phi(t, x)=p \cdot x-\phi(t, x)$ then (27) holds. For small $\delta>0$, we choose $\phi(t, x):=p \cdot x+(q \cdot x)^{2}+\delta\left(|x|^{2}+(t-1)^{2}\right)$ and observe that $(1,0)$ is a strict maximum of $u(t, x)-\phi(t, x)$ for any $\delta$; moreover (27) reads

$$
-A(p) q \cdot q=-\operatorname{tr}[A(p)(q \otimes q)] \leq o(\delta)
$$

where $o(\delta) \rightarrow 0$ as $\delta \rightarrow 0$. Since the inequality $A(p) q \cdot q \geq 0$ holds for any $p$ and $q$, the thesis follows letting $\delta \rightarrow 0$.

Step $4\left(\overline{\mathbf{u}}(\mathbf{x}, \mathbf{0})=\underline{\mathbf{u}}(\mathbf{x}, \mathbf{0})=\mathbf{u}_{\mathbf{0}}(\mathbf{x})\right.$.) Let $v_{0}$ be the function appearing in (25). For every $\delta>0$, it is possible to find $v_{+}^{\delta}, v_{-}^{\delta} \in \mathcal{C}^{\infty}\left(\mathbb{R}^{n}\right) \cap W^{2, \infty}\left(\mathbb{R}^{n}\right)$ such that $v_{-}^{\delta} \leq v_{0} \leq v_{+}^{\delta}$ and that $\| v_{+}^{\delta}-$ $v_{0}\left\|_{\infty},\right\| v_{-}^{\delta}-v_{0} \|_{\infty} \leq \delta$ (this can be done by using standard mollification arguments). For any fixed $\delta>0$, we consider the functions

$$
w_{ \pm}^{\varepsilon, \delta}(t, x):=v_{ \pm}^{\delta}(x)+q \cdot x+\varepsilon \chi_{ \pm}\left(\frac{x}{\varepsilon}\right) \pm\left(C^{\delta} t+\varepsilon\left\|\chi_{ \pm}\right\|_{\infty}\right)
$$

where $\chi_{+}(x)=\chi\left(x ; q+D v_{+}^{\delta}(x)\right)$ is the solution to (13) with $p=q+D v_{+}^{\delta}(x), \chi_{-}(x)=\chi\left(x ; q+D v_{-}^{\delta}(x)\right)$ is the solution to (13) with $p=q+D v_{-}^{\delta}(x)$, and $\chi_{ \pm}(0)=0$. Choosing $C^{\delta}>0$ sufficiently large, it is easy to see that $w_{ \pm}^{\varepsilon, \delta}$ are respectively a super and a subsolution to (24). Moreover $w_{-}^{\varepsilon, \delta}(0, x) \leq u_{0}(x) \leq w_{+}^{\varepsilon, \delta}(0, x)$. So, by the comparison principle (Theorem 5.1]), we get that $w_{-}^{\varepsilon, \delta}(t, x) \leq u^{\varepsilon}(t, x) \leq w_{+}^{\varepsilon, \delta}(t, x)$, for every $\delta>0$.

Passing to the relaxed semilimits, we then obtain

$$
v_{-}^{\delta}(x)+q \cdot x \leq \underline{u}(0, x) \leq \bar{u}(0, x) \leq v_{+}^{\delta}(x)+q \cdot x .
$$

Letting $\delta \rightarrow 0$, this gives $\bar{u}(x, 0)=\underline{u}(x, 0)=u_{0}(x)$.

Step 5 (Uniform convergence.) Let us define $\bar{v}(t, x):=\bar{u}(t, x)-q \cdot x$ and $\underline{v}(t, x):=\underline{v}(t, x)-q \cdot x$. It is easy to show that $\bar{v}$ and $\underline{v}$ are bounded, satisfy $\bar{v}(0, x)=v_{0}(x)=\underline{v}(0, x)$ and are respectively a sub and a supersolution of

$$
v_{t}(t, x)=\operatorname{tr}\left[A(q+D v(t, x)) D^{2} v(t, x)\right] \quad \text { in }(0,+\infty) \times \mathbb{R}^{n} .
$$

Then, by the comparison principle (Theorem 5.2), we obtain that $\bar{v}(t, x) \leq \underline{v}(t, x)$ in $(0,+\infty) \times \mathbb{R}^{n}$, and therefore $\underline{v}(t, x)=\bar{v}(t, x)$ in $(0,+\infty) \times \mathbb{R}^{n}$, since the opposite inequality holds by definition of 
the semilimits. In particular, we get that $v(t, x):=\bar{v}(t, x)=\underline{v}(t, x)$ is the unique bounded viscosity solution to (29) with initial data $v_{0}$.

This implies that $\bar{u}(t, x)=\underline{u}(t, x)=u(t, x)$ is the unique continuous viscosity solution to (26) in the class $\mathcal{L}_{q}$, with initial datum $u_{0}$. Moreover, using the definition of relaxed limits, we obtain that $u^{\varepsilon}(t, x) \rightarrow u(t, x)$ locally uniformly.

We observe that Theorem 5.3 has also a geometric counterpart. Namely, given a solution $u^{\varepsilon}$ to (24) we consider its graph $\Gamma^{\varepsilon}(t):=\left\{\left(x, x_{n+1}\right) \in \mathbb{R}^{n+1}: x_{n+1}=u^{\varepsilon}(x, t)\right\}$. Then, $\Gamma^{\varepsilon}(t)$ evolves in time accordingly to (2). In the limit $\varepsilon \rightarrow 0$, we then obtain the geometric evolution

$$
V=\alpha(\nu) H
$$

where the function $\alpha$ is defined as

$$
\alpha(\nu):=\frac{A\left(-\nu_{x} / \nu_{n+1}\right)}{\nu_{n+1}^{2}} \geq 0 \quad \nu_{n+1} \neq 0 .
$$

Note that, when $n=1$, due to (20), we have $0<\alpha(\nu) \leq K_{g}\left|\nu_{2}\right|$, which implies in particular $\lim _{\nu_{2} \rightarrow 0} \alpha(\nu)=0$.

As discussed for instance in 21, the asymptotic limit of (2) is strictly related to the existence of compact embedded solutions to the following prescribed curvature problem:

$$
H+g=0 .
$$

Indeed, the existence of a compact solution to (31) implies by rescaling the existence of compact solutions to

$$
H+\frac{1}{\varepsilon} g\left(\frac{x}{\varepsilon}\right)=0,
$$

which can be used as barriers for the evolution (2). It would then follow that the solutions to (2) converge, as $\varepsilon \rightarrow 0$, to a stationary hypersurface, thus implying $\alpha \equiv 0$ in (30), that is, $A(p)=0$ for all $p \in \mathbb{R}^{n}$.

However, if $g$ has zero average and does not depend on $x_{n+1}$, from Remark 3 we know that $A(0)>0$ if $\|g\|_{\text {Lip }}<\delta$; moreover we know from (20) that $A(p)>0$ for all $p$, if $n=1$ and $g$ satisfies (12). As a consequence, in such cases we conclude that there are no compact embedded solutions to (31).

We point out that this result cannot be expected for a generic function $g$, which is periodic and of zero average, but which depends also on $x_{n+1}$. Indeed, in 21] it has been proved that we can always find a sequence $g_{n} \rightarrow g$ in $L^{1}\left(\mathbb{R}^{n}\right)$, where the functions $g_{n}$ are all periodic, of zero average, uniformly bounded, and satisfy (11), such that there exist compact embedded solutions to (31) with $g$ replaced by $g_{n}$.

Acknowledgements. The second and the third authors wish to thank the University of Tours and the Research Institute le Studium for the kind hospitality and support.

\section{References}

[1] O. Alvarez, M. Bardi. Ergodicity, stabilization, and singular perturbations for Bellman-Isaacs equations. Mem. Amer. Math. Soc., 204(960), 2010.

[2] L. Alvarez, F. Guichard, P.L. Lions and J.M. Morel. Axioms and fundamental equations of image processing. Arch. Rational Mech. Anal., 123(3):199-257, 1993.

[3] L. Ambrosio, N. Fusco, D. Pallara. Functions of Bounded Variation and Free Discontinuity Problems. Oxford Mathematical Monographs, 2000. 
[4] M. Arisawa, P.-L. Lions. On ergodic stochastic control. Comm. Partial Differential Equations, 23(11-12):2187-2217, 1998.

[5] G. Barles, S. Biton, M. Bourgoing, O. Ley. Uniqueness results for quasilinear parabolic equations through viscosity solutions methods. Calc. Var., 18:159-179, 2003.

[6] G. Barles and P.E. Souganidis. A new approach to front propagation problems : theory and applications. Arch. Rat. Mech. Anal., 141:237-296, 1998.

[7] A. Bensoussan. Perturbation methods in optimal control. John Wiley \& Sons, Ltd., Chichester, Gauthier-Villars, Montrouge, 1988.

[8] S. Biton. Nonlinear monotone semigroups and viscosity solutions. Annales IHP - Analyse Nonlinéaire, 18(3):383-402, 2001.

[9] L. A. Caffarelli, R. de la Llave. Planelike minimizers in periodic media. Comm. Pure Appl. Math., 54(12):1403-1441, 2001.

[10] P. Cardaliaguet, P.-L. Lions, P. E. Souganidis. A discussion about the homogenization of moving interfaces. J. Math. Pures Appl., 91:339-363, 2009.

[11] A. Cesaroni, M. Novaga, E. Valdinoci. Curvature flow in heterogeneous media. Preprint, 2010.

[12] B. Craciun, K. Bhattacharya. Effective motion of a curvature-sensitive interface through a heterogeneous medium. Interfaces Free Bound., 6:151-173, 2004.

[13] M.G. Crandall, H. Ishii, P.-L. Lions. Users guide to viscosity solutions of second order partial differential equations, Bull. Amer. Math. Soc., 27:1-67, 1992.

[14] N. Dirr, G. Karali, N. K. Yip. Pulsating wave for mean curvature flow in inhomogeneous medium. European J. Appl. Math., 19:661-699, 2008.

[15] L. C. Evans. The perturbed test function method for viscosity solutions of nonlinear PDE. Proc. Roy. Soc. Edinburgh Sect. A, 111:359-375, 1989.

[16] E. Giusti. On the equation of surfaces of prescribed mean curvature. Invent. Math., 46(2):111137, 1978.

[17] D. Gilbarg, N. Trudinger. Elliptic partial differential equations of second order. Classics in Mathematics, Springer-Verlag, Berlin, 2001.

[18] S. Kirsch, P. Laurain. An obstruction to the existence of immersed curves of prescribed curvature. Potential Anal., 32(1):29-39, 2010.

[19] P.-L. Lions, P. E. Souganidis. Homogenization of degenerate second-order PDE in periodic and almost periodic environments and applications. Annales IHP - Analyse Nonlinéaire, 22(5):667-677, 2005.

[20] A. Lunardi. Analytic semigroups and optimal regularity in parabolic problems. Progress in Nonlinear Differential Equations and their Applications, 16, Birkhäuser Verlag, Basel, 1995.

[21] M. Novaga, E. Valdinoci. Bump solutions for the mesoscopic Allen-Cahn equation in periodic media. Calc. Var. PDE, to appear.

[22] A. Ros. The isoperimetric problem. In Global theory of minimal surfaces, Clay Math. Proc., 2:175-209, Amer. Math. Soc., 2005. 